\title{
OTIMIZAÇÃO DE PROCESSO NO LAMINADOR DE ENCRUAMENTO REVERSÍVEL DA CSN*
}

\author{
Raffaele Alves Paranhos ${ }^{1}$ \\ Rafael Masulck Santos ${ }^{2}$ \\ Vitor Tomaz de Aquino ${ }^{1}$ \\ Wellington Egmar da Silva ${ }^{3}$ \\ Eszenrral Correa Duarte ${ }^{3}$ \\ Odilei Max da Silva ${ }^{3}$
}

\section{Resumo}

Devido à alta competitividade no mercado siderúrgico se faz necessário à otimização do processo como forma a atendermos a demanda de produção com um menor custo de transformação. Nesse contexto, foi utilizado para desenvolvimento do trabalho a metodologia DMAIC e os conceitos do "LEAN". O objetivo do grupo foi estratificar o custo de produção do equipamento e atuar nos principais fatores, tendo como princípio a redução do desperdício, aumento da produtividade da mão de obra, aliada a produtividade de tonelagem de material. Na estratificação do custo de produção identificamos o "Rendimento metálico" como sendo o fator mais significativo e propomos ações internas que contemplaram alteração de programa de automação para melhora do descarte da cauda (Final) da bobina e com isso atingindo valores recordes de aproveitamento material acabado. Fizemos reduções de lead time do nosso processo eliminando os tempos que não agregam valores, aumentando a produtividade do equipamento. Com este trabalho conseguimos reduzir consideravelmente o custo de transformação do equipamento, além, de aumentar sua capacidade produtiva.

Palavras-chave: Produtividade; Custo; Setup.

\section{Abstract}

\section{PROCESS OPTIMIZATION IN TEMPER MILL CSN}

Due to the high competitiveness in the steel market in the world, is necessary to optimize the production process in order to commit the production demand at a lower cost. In this context, was used to develop this work DMAIC tools and its concepts of "lean manufacturing". The aim was to stratify the equipment production costs and act on the main factors having as principle the reduce waste, increase the labor productivity, together with the productivity of material tonnage. When stratifying the production costs we identified the "metallic yield" as the most significant factor. So we made internal plans that consider change automation program to reduce the coil tail disposal (end) reaching with this action records of final material usage. We reduce the lead time process eliminating times that do not add value, increasing equipment productivity. This work significantly reduced the equipment process costs besides its production capacity.

Keywords: Productivity; Cost; Setup; Automation.

1 Eng. de Manutenção, Companhia Siderúrgica Nacional, Volta Redonda, RJ, Brasil.

2 Eng. Metalurgista, Companhia Siderúrgica Nacional, Volta Redonda, RJ, Brasil.

3 Tec. Metalurgista, Companhia Siderúrgica Nacional, Volta Redonda, RJ, Brasil. 


\section{INTRODUÇÃO}

O processo de bobina no Laminador de Encruamento Reversível da CSN (LER) tem como objetivo eliminar o patamar de escoamento através de um pequeno alongamento do material, melhorar o acabamento superficial ajustando o nível de propriedade mecânico desejado [1], obtendo-se assim o produto BFF (Bobina Fina a Frio) via Tratamento térmico nos Fornos de Recozimento em Caixa (FRCX).

Para tornar o produto BFF mais competitivo, se faz necessário à análise do custo de transformação do produto como forma de otimizar o processo de encruamento.

O Laminador de Encruamento Reversível é do tipo "Six high" e possui peso máximo de entrada de 25 toneladas, cujos materiais são para clientes da linha automobilística, linha branca e uso geral.

Processa material de diversas especificações (desde a especificação de SAE até matérias IF), com espessuras variando entre 0,30 a $3,0 \mathrm{~mm}$ e largura de 700 a $1570 \mathrm{~mm}$.

\section{MATERIAIS E MÉTODOS}

Utilizando a filosofia de trabalho que tem como foco o conceito de "fazer mais com menos" e, principalmente, "Eliminando os desperdícios" e "Tempos que não agregam valores ao produto (TNAV)" [2], foi feita a estratificação do nosso custo de transformação para buscarmos as melhorias no processo, de forma a controlar e reduzi-los.

Conforme já mencionado, este trabalho teve como base o uso da ferramenta DMAIC, que é usada em projetos focados em melhorar processos de negócios já existentes, sendo dividido em cinco etapas: Define (Definir), Measure (Medir), Analyze (Analisar), Improve (Melhorar) e Control (Controlar), conforme será retratado ao logo dessa dissertação [2].

\subsection{Fase Define}

Após estratificação e análise detalhada de todos os itens que geram impacto no custo de transformação do equipamento, foram definidas as "Metas especificas" [2], necessárias para o objetivo de "Otimizar o processo de Encruamento":

- Aumento do Rendimento metálico a partir da redução da perda metálica gerada (Diferença percentual entre o peso de entrada e peso de saída do equipamento) em $0,4 \%$. Vide figura 1 os detalhes referentes ao cálculo que definem o foco 1 .

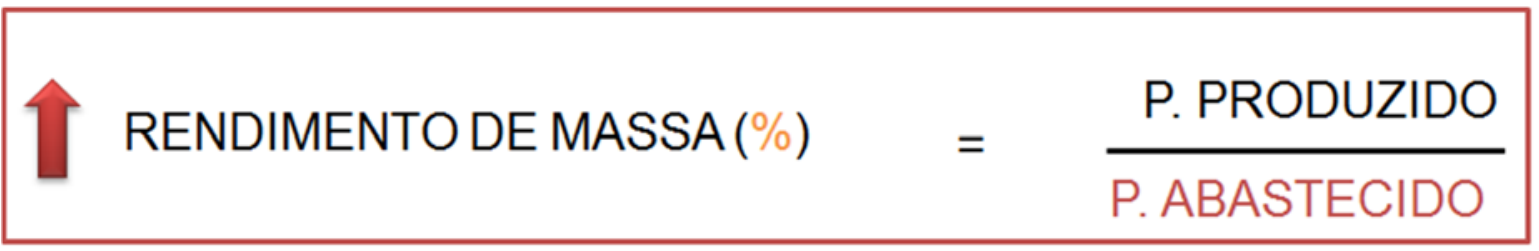

Figura 1. Calculo do "Rendimento de massa (\%)". Peso produzido ("P. Produzido") dividido pelo peso abastecido ("P. Abastecido"). Representa a capacidade do equipamento em evitar a perda de material. 
- Aumento produtividade mão de obra (Homens por Tonelada Produzida) em $4 \%$. Vide figura 2 os detalhes referentes ao cálculo que definem o foco 2.

\section{PRODUTIVIDADE (Ton/HOMEM) = PRODUÇÃO \\ COLABORADOR}

Figura 2. Calculo de "Produtividade da mão de obra (ton/Homem)". Peso produzido ("Produção") dividido pelo numero de colaboradores necessários ("Colaborador"). Representa a capacidade de o equipamento produzir com determinado número de colaboradores.

- Aumento da produtividade do equipamento (Tonelada produzida por Hora) em $10 \%$. Vide na figura 3 os detalhes referentes ao cálculo que definem o foco 3 .

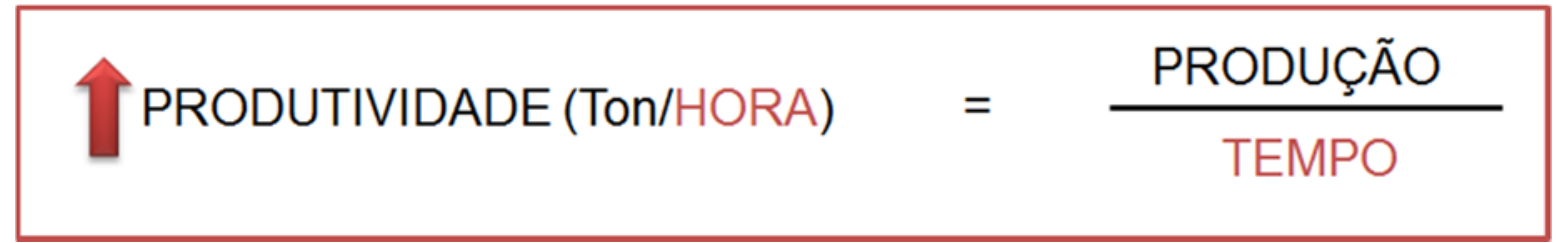

Figura 3. Calculo de "Produtividade (ton/Hora)". Peso produzido ("Produção") dividido pelo tempo necessário ("Tempo"). Representa a capacidade do equipamento em produzir em um determinado tempo.

\subsection{Fase Measure}

Foram executadas medições relacionadas a cada uma das 3 "metas especificas" visando identificar os "Focos do Problema" [3], sendo eles:

Foco 1- Redução tempo de ciclo

Foco 2- Redução de parada por troca de diâmetro

Foco 3- Otimização dos postos operacionais

Foco 4- Redução das perdas metálicas na cauda

\subsubsection{Produtividade}

Após análise do diagrama de estado destacado na Figura 4, foi identificado a necessidade de atuar na redução dos tempos de ciclo (Foco $n^{\circ} 1$ ), reduzindo os tempos que não agregam valor ao processo

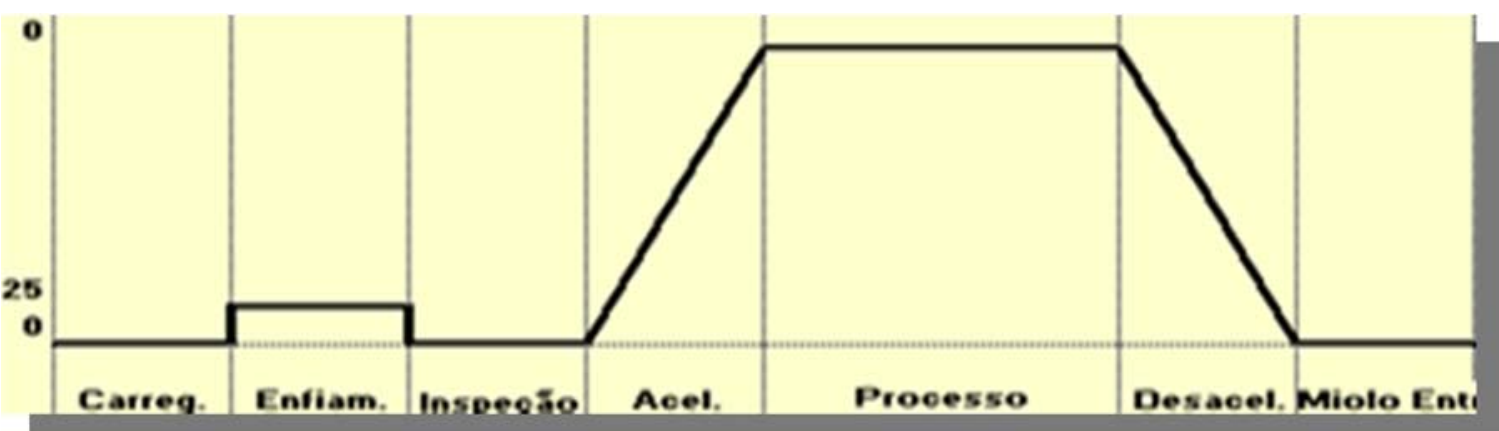

Figura 4. Diagrama de estado, representando o Tempo de ciclo ("Eixo X") em cada etapa de processo. 
Estratificando o total de tempo de parada do equipamento foi identificada a necessidade de reduzir as paradas por "Troca de diâmetro interno da bobina" (Foco $\mathrm{n}^{\circ} 2$ ), onde esta correspondia a $18 \%$ de todas as paradas. Vide análise completa na Figura 5 abaixo.

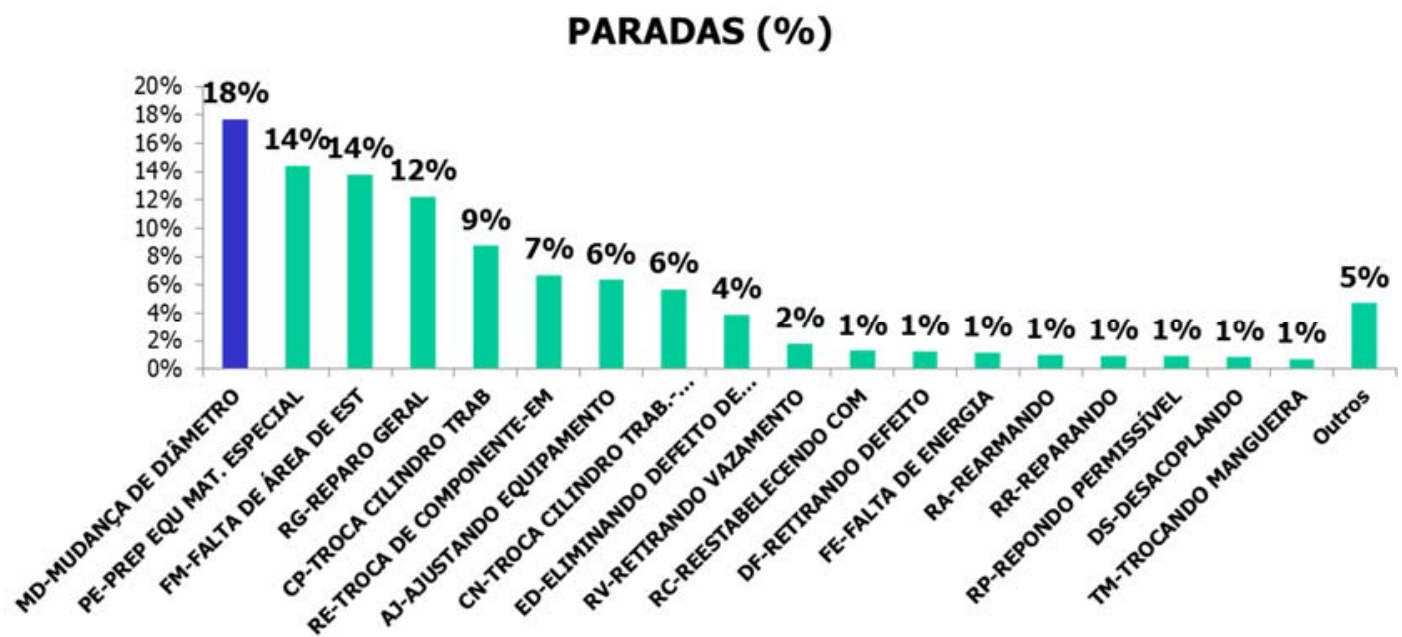

Figura 5. Estratificação do "Tempo de equipamento parado". Evidencia a causa de maior tempo de equipamento parado.

\subsubsection{Produtividade mão de obra}

Após estudo de "Tempos e movimentos" destacado na figura 6 abaixo, foi constatado a necessidade de "Otimizar os postos operacionais" de forma a encontrar oportunidades de produzir com um efetivo menor (Foco $n^{\circ} 3$ ).

A partir da avaliação de todas as funções dos operadores medidos através do diagrama de estados, conseguimos identificar a necessidade de relocar equipamentos e funções operacionais de formar a eliminar a função "operador de piso".

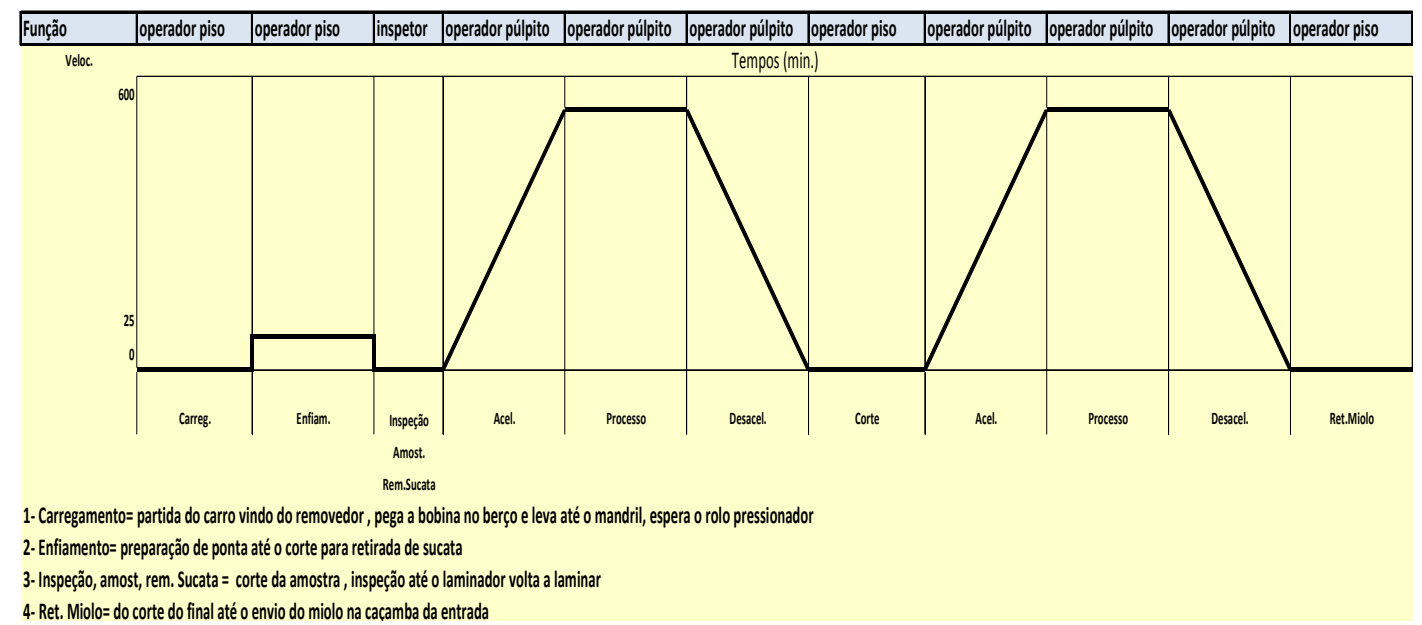

Figura 6. Diagrama de estado, correlacionando a relação entre as funções de processo (Destacado na parte superior do gráfico) com as etapas de processo ( Destacado na parte inferior do gráfico).

\subsubsection{Redução da perda metálica}

Feito estratificação das principais perdas metálicas do processo e identificado que havia um descarte excessivo de fora de bitola. Com isso, foi observado e identificado 
onde se poderia atuar dentro do processo, visto que o fora de bitola é um defeito inerente ao processo.

Após visita in loco identificou-se um descarte a mais na cauda acima do fora de bitola estabelecido pelo Laminador de tiras a frio 3 , devido ao operador não ter recurso de identificação na entrada do laminador de encruamento e também de não poder fazer a laminação deste trecho sem tensão após o corte. A partir daí surgiu a necessidade de estudar e ter a criatividade para desenvolver uma função nova na automação para uso da operação (Foco n4).

\subsection{Fase Analyse}

Após definição dos "4 focos" a serem trabalhados, foi feito um brainstorning através de diagrama de causa e efeito e obtidos 30 "Causas potenciais", e priorizou-se estas causas utilizando a matriz GUT destacada na figura 7.

\begin{tabular}{|c|c|c|c|c|c|c|c|c|c|c|}
\hline \multicolumn{11}{|c|}{ MATRIZ DE PRIORIZAÇÃO E CONSENSO } \\
\hline \multirow[t]{2}{*}{ Critérios de Priorização } & \multicolumn{3}{|c|}{ GRAVIDADE } & \multicolumn{3}{|c|}{ URGÊNCIA } & \multicolumn{3}{|c|}{ TENDÊNCIA } & 0 \\
\hline & \multicolumn{3}{|c|}{$\begin{array}{l}\text { Qual o nivel da gravidade do problema, } \\
\text { está causando muito prejuizo, acidentes, } \\
\text { desmotivacao,etc? }\end{array}$} & \multicolumn{3}{|c|}{$\begin{array}{l}\text { Qual o nivel da urgencia da solucao em se } \\
\text { resolver isto? Há muita pressa ou pode se } \\
\text { esperar um pouco? }\end{array}$} & \multicolumn{3}{|c|}{$\begin{array}{l}\text { Qual a tendência de piorar se nada for } \\
\text { fetito agora? }\end{array}$} & T \\
\hline \multirow[t]{2}{*}{$\begin{array}{c}\text { Causas fundamentais do } \\
\text { problema }\end{array}$} & $\begin{array}{l}\text { Muito } \\
\text { Grave }\end{array}$ & $\begin{array}{l}\text { Gravidade } \\
\text { Média }\end{array}$ & $\begin{array}{l}\text { Sem } \\
\text { Gravidade }\end{array}$ & $\begin{array}{l}\text { Muito } \\
\text { Urgente }\end{array}$ & $\begin{array}{l}\text { Média } \\
\text { Urgência }\end{array}$ & $\begin{array}{c}\text { Sem } \\
\text { Urgência }\end{array}$ & $\begin{array}{l}\text { Muita } \\
\text { tendência }\end{array}$ & $\begin{array}{l}\text { Média } \\
\text { tendência }\end{array}$ & $\begin{array}{c}\text { Sem } \\
\text { tendência }\end{array}$ & A \\
\hline & 5 & 3 & 1 & 5 & 3 & 1 & 5 & 3 & 1 & \\
\hline 1. Posicionamento do Carro no carregamento & & $\bar{x}$ & & $x$ & & & $x$ & & & 13 \\
\hline 2. Perda de tempo no descarregamento & & $x$ & & $x$ & & & $x$ & & & 13 \\
\hline 3. Falta de monitoramento dos tempos de processo & $\mathrm{x}$ & & & & $\mathrm{x}$ & & $\mathrm{x}$ & & & 13 \\
\hline 4. Elevado número de diâmetro 20 entrega direta & $x$ & & & & $x$ & & $x$ & & & 13 \\
\hline 5.Falta de controle do estoque intermediário & 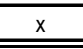 & & & $x$ & & & 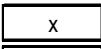 & & & 15 \\
\hline 6.Falta de comandos operacionais no púlpito & $x$ & & & & $\mathrm{x}$ & & $\overline{\mathrm{x}}$ & & & 13 \\
\hline 7.Excesso de descartes na cauda & $x$ & & & & $\bar{x}$ & & $\bar{x}$ & & & 13 \\
\hline
\end{tabular}

Figura 7. Ferramenta de priorização "Diagrama de causa e efeito".

Após o uso da ferramenta "Matriz GUT", foi possível selecionar as 5 causas a serem priorizadas, nomeando-as de "Causas Fundamentais" [3].

Essas "Causas fundamentais", que são relacionadas com os 4 "Focos" do projeto, conforme figura 8 abaixo.

\begin{tabular}{|c|c|c|c|c|c|}
\hline CAUSA & FOCO 1 & FOCO 2 & FOCO 3 & FOCO 4 & CAUSA FUNDAMENTAL \\
\hline 1 & $\mathrm{X}$ & & & & $\begin{array}{r}\text { Posicionamento / sequenciamento do carro transportador de } \\
\text { bob, gerando perda de TC. }\end{array}$ \\
\hline 2 & $\mathrm{X}$ & & & & Falta de monitoramento dos tempos do processo \\
\hline 3 & & $\mathrm{X}$ & & & Falta de controle do estoque intermediário \\
\hline 4 & & & $\mathrm{X}$ & & Falta de comandos no púlpito e distribuição das funções \\
\hline 5 & & & & $\mathrm{X}$ & Excesso de descartes na cauda \\
\hline
\end{tabular}

Figura 8. Correlação das 5 "Causas Fundamentais" com os 4 "Focos" do trabalho. 


\subsection{FASE IMPROVE}

Visando implementar as 5 ações, referentes as 5 "Causas fundamentais", foi desenvolvido um extenso plano de ação, envolvendo diversos setores, sendo implementado $100 \%$ do planejado. Detalhes do plano de ação podem ser avaliados na figura 9 abaixo.

\begin{tabular}{|c|c|c|c|c|}
\hline O que? & Como? & Por que? & Quem? & Foco \\
\hline $\begin{array}{c}\text { Criar automação } \\
\text { para redução de } \\
\text { tempo de ciclo }\end{array}$ & $\begin{array}{c}\text { Alterando } \\
\text { programação do } \\
\text { LER }\end{array}$ & $\begin{array}{c}\text { Reduzir TNAV no } \\
\text { processo }\end{array}$ & Grupo & $\begin{array}{c}\text { Prod } \\
\text { (thora) }\end{array}$ \\
\hline $\begin{array}{c}\text { Criar telas no } \\
\text { sistema para } \\
\text { controle estoque } \\
\text { intermediário }\end{array}$ & $\begin{array}{c}\text { Desenvovendo as } \\
\text { telas de acordo } \\
\text { com os controle } \\
\text { atuais em } \\
\text { planilha }\end{array}$ & $\begin{array}{c}\text { Para melhorar } \\
\text { controle do } \\
\text { estoque frio do } \\
\text { LER }\end{array}$ & Grupo & $\begin{array}{c}\text { Prod } \\
\text { (t/hora }\end{array}$ \\
\hline $\begin{array}{c}\text { Automatizar } \\
\text { controle de } \\
\text { ponta e cauda } \\
\text { no descarte }\end{array}$ & $\begin{array}{c}\text { Instalando via } \\
\text { PLC, lógica de } \\
\text { corte e parada } \\
\text { automática do } \\
\text { Laminador }\end{array}$ & $\begin{array}{c}\text { Para evitarmos } \\
\text { descarte de ponta } \\
\text { e cauda dentro } \\
\text { de tolerância do } \\
\text { cliente }\end{array}$ & Grupo & RM(\%) \\
\hline $\begin{array}{c}\text { Automatizar } \\
\text { funções e } \\
\text { operações do } \\
\text { laminador }\end{array}$ & $\begin{array}{c}\text { Automatizando } \\
\text { algumas } \\
\text { operações e } \\
\text { distribuindo as } \\
\text { funções. }\end{array}$ & $\begin{array}{c}\text { Para garantir } \\
\text { possibilidade de } \\
\text { operação com } \\
\text { contingente } \\
\text { menor }\end{array}$ & Grupo & Prod \\
\hline
\end{tabular}

Figura 9. Resumo do "Plano de ação" desenvolvido pela equipe, com o objetivo de atingir a "Meta Global".

\section{IMPLEMENTAÇÃO}

\subsection{Criar Automação para Reduçãod Tempo de Ciclo}

Um diagrama de transição de estados [4] é uma representação do estado ou situação em que um objeto pode se encontrar no decorrer da execução de processos de um sistema. Com este método pode-se fazer o levantamento de todos os estados que o objeto se encontra, neste caso denomina-se objeto o estado em que o laminador se encontra e então contar o tempo até a sua próxima transição. $\mathrm{Na}$ figura 10 abaixo, é possível observar o diagrama de estado elaborado para este intento. 


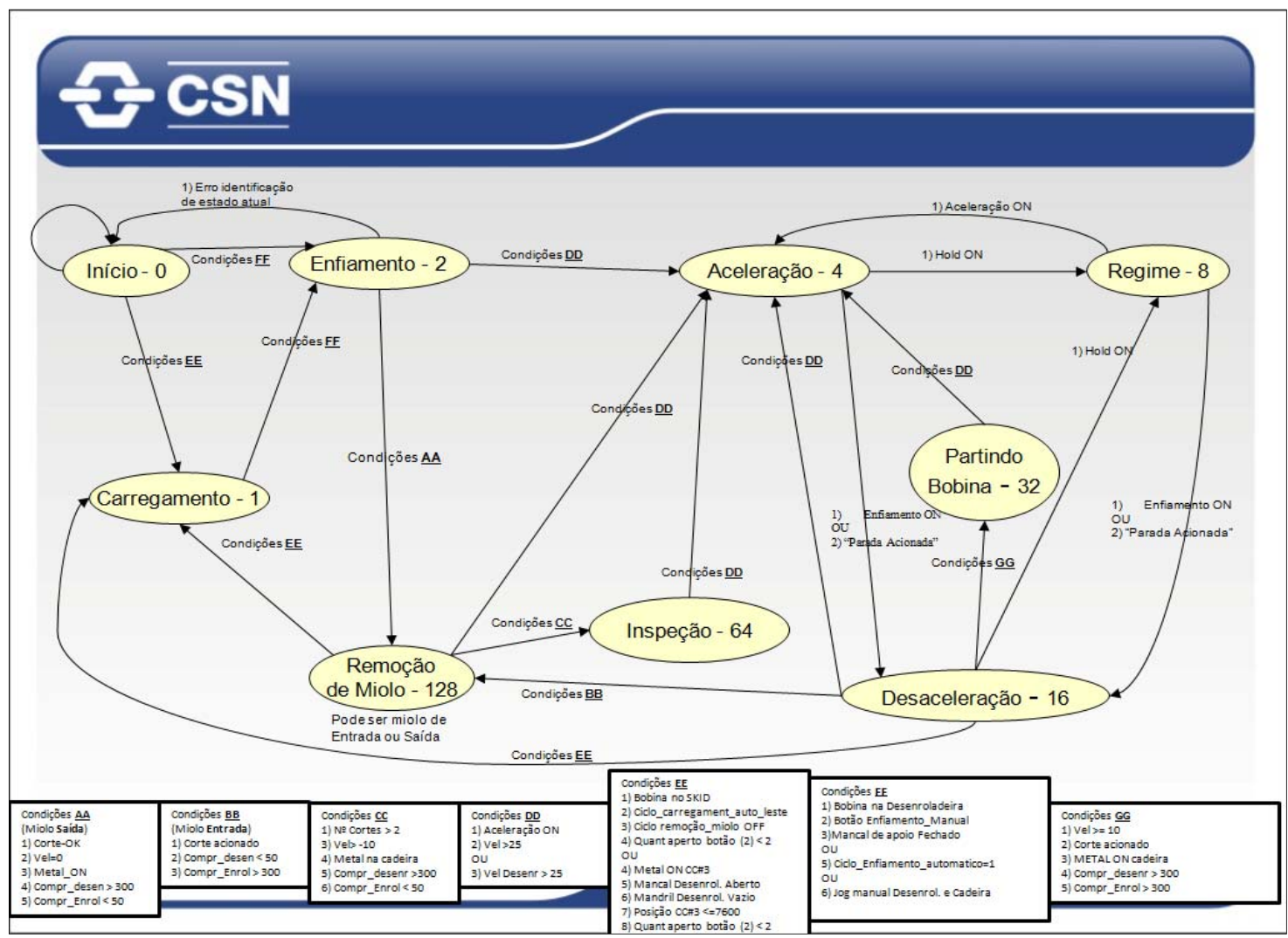

Figura 10. Diagrama de estados do laminador.

\subsubsection{Lógica no CLP}

Para implantação da lógica do diagrama de estados no CLP (Controlador Lógico Programável) utilizado, teve-se que encontrar em sua biblioteca blocos livres de funções de intertravamento lógico, blocos de funções aritméticas de alta performance, além de blocos temporizadores com função interna de contadores de tempo.

O programa foi montado de forma que apenas um estado fosse possível por vez, ou seja, todas as condições da transição que antecede o estado fossem verdadeiras para que houvesse a transição de um estado para o outro. Nunca dois estados podem acontecer juntos.

Assim que uma transição de estado acontece e entra em um determinado estado, um temporizador é "disparado" fazendo a contagem de tempo daquele estado, até que nova transição aconteça. Assim, este tempo é preservado neste temporizador até que todo o ciclo do processo aconteça e todos os temporizadores sejam zerados simultaneamente. Veremos a diante que antes de zerar o contador estes valores de tempo de cada estado são armazenados para consulta em relatório.

\subsubsection{Sistema supervisório}

Para que os tempos de cada estado pudessem ser acompanhados pelas pessoas, cada temporizador que acumula o tempo de cada estado teve que ser disponibilizado por uma "TAG" em uma tela do sistema supervisório do equipamento.

\subsubsection{Relatórios dos tempos}

Após armazenar os dados de cada bobina por estados e suas justificativas de tempos armazenados em um único arquivo, utilizamos o software Excel para 
elaboração de relatórios de tempos que relacionam o desempenho por equipe, por estado durante o período que o usuário determinar, além de um relatório diário que relaciona os problemas do dia assim como o desempenho por equipe.

Os relatórios foram elaborados utilizando o VBA do Excel, cuja algumas referências se fizeram necessárias para utilização de uma planilha do Excel como banco de dados. Importamos o arquivo .txt para dentro do Excel. Tratamos os dados e utilizamos comandos SQL para geração de relatórios;

Existem algumas limitações quanto ao uso destas funções, mas no nosso caso não tivemos muitos problemas para elaboração deste intento [5].

\subsection{Criar Controle para Ponta e Cauda no Descarte}

O processo de descarte da ponta e cauda da bobina no laminador se dá através do desenrolar da ponta da bobina na desenroladeira, depois o corte na saída para descarte do fora de bitola e a retirada deste pelo carro transportador.

O descarte da cauda é feito quando se finaliza a laminação da bobina e a cauda é cortada na tesoura de saída do laminador, depois é enrolada pela desenroladeira e feito descarte pela entrada. O problema era que ambos os cortes eram feitos na saída.

Após ótimo trabalho de restabelecimento do raio-x de entrada que apresentava muitos problemas, foi possível fazer a verificação da espessura da tira na entrada e com isso executar o corte também na entrada.

Então, se fez necessária implantação de nova lógica de programação no CLP para que ao se acionar a tesoura de entrada e o processo de laminação ocorresse, a cadeira deve manter uma força constante e a laminação da tira que ainda não tinha sido laminada fosse aproveitada. Para isso, a velocidade deverá parar exatamente na "mordida" dos cilindros. Assim seria possível fazer o aproveitamento de material de oito metros a cada bobina.

\subsection{Automatizar Funções e Operações no Laminador}

A fim de aumentar a produtividade da mão de obra se fez necessário um levantamento das funções de operação baseado na experiência da equipe que nele trabalha identificando os comandos "chaves" para tal.

Depois de algumas reuniões com as equipes chegamos à conclusão de que algumas mudanças nos comandos seriam necessárias.

Para a implementação deste escopo foi necessário grande planejamento para preparação de compra e de montagem de botões na mesa de operação existente, implantação de nova IHM com programação de novos comandos no púlpito, passagem de novos cabos, teste e implantação da nova lógica no PLC.

\section{RESULTADOS E DISCUSSÕES}

Após a implementação das ações tivemos a obtenção das metas específicas.

- Redução da perda metálica gerada (Diferença percentual entre o peso de entrada e peso de saída do equipamento).

Foi observado aumento do rendimento metálico de $0,7 \%$, superando a meta especifica de $0,4 \%$.

- Aumento da produtividade de mão de obra (Homens por Tonelada Produzida). 
Foi observado aumento de $24 \%$ na produtividade da mão de obra do equipamento, superando a meta especifica de $4 \%$.

- Aumento da produtividade do equipamento (Tonelada produzida por Hora).

Foi observado aumento de $12 \%$ na produtividade do equipamento, superando a meta especifica de $10 \%$.

\section{CONCLUSÃO}

Conseguimos atingir nosso objetivo de otimizar o processo, de forma a reduzir o custo de transformação, e com isso aumentando a competitividade do produto. Tivemos com esse trabalho um grande aprendizado em relação ao conceito de Gestão do processo e possibilitando a disseminação das ações tomadas em outros equipamentos da CSN.

\section{Agradecimentos}

Os autores agradecem à CSN por permitir a publicação do presente trabalho e a todas as pessoas engajadas na realização deste que não puderam figurar como coautores (equipes de laboratórios, de produção, área técnica e desenvolvimento de produtos).

\section{REFERÊNCIAS}

1 Padilha, A. F. e Siciliano Jr, F.. Encruamento, Recristalização, Crescimento de Grão e Textura. $3^{a}$ Edição.São Paulo: ABM. 2005.

2 Werkema, Cristina, Criando a Cultura Seis Sigma. $4^{\text {a }}$ Edição. Belo Horizonte, MG: Editora Werkema. 2010.

3 Werkema, Cristina , Avaliação de sistemas de Medição. $5^{a}$ Edição. Belo Horizonte, MG: Editora Werkema. 2006.

4 Wikipedia, Diagrama de Transição de estado, http://pt.wikipedia.org/wiki/Diagrama_de_transi\%C3\%A7\%C3\%A3o_de_estados, 09/03/2015

5 Ambiente Office, Manipular uma pasta de trabalho através de uma conexão ADO, http://www.ambienteoffice.com.br/excel/manipular_uma_pasta_de_trabalho_atraves_de _uma_conexao_ado, 09/03/2015 